\title{
Development of genomic simple sequence repeat markers for Glycyrrhiza lepidota and cross-amplification of other Glycyrrhiza species
}

Jun Hyoung Bang ${ }^{1}$, Chi Eun Hong ${ }^{2}$, Sebastin Raveendar ${ }^{3}$, Kyong Hwan Bang ${ }^{2}$, Kyung Ho Ma ${ }^{2}$, Soon Wook Kwon ${ }^{4}$ , Hojin Ryu ${ }^{5}$, Ick Hyun Jo ${ }^{\text {Corresp., } 2}$, Jong Wook Chung ${ }^{\text {Corresp. } 1}$

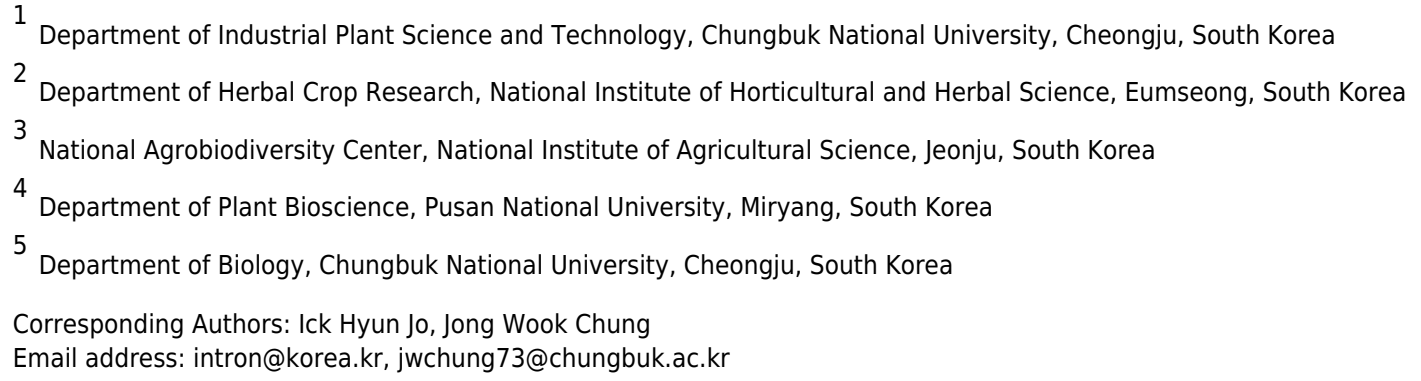

Background. Licorice (Glycyrrhiza spp. L. ) is used as a natural sweetener and medicinal herb in European and Asian countries . Molecular studies have been conducted to find differences between wild and cultivated species because most wild species are highly resistant to abiotic and biotic stresses compared with their cultivated species. However, few molecular markers have been developed for studying the genetic diversity and population structure of licorice species and to identify differences between cultivars. Thus, the present study aimed to develop a set of genomic simple sequence repeat (SSR) markers for molecular studies of these species.Methods. In the present study, we developed polymorphic SSR markers based on whole-genomesequence data of Glycyrrhiza lepidota. Then, based on the sequence information, the polymorphic SSR markers were developed. The SSR markers were applied to 23 Glycyrrhiza individual plants. We also evaluated the phylogenetic relationships and interspecies transferability among samples. Results. The genetic diversity analysis using these markers identified 2-23 alleles, and the major allele frequency, observed heterozygosity, genetic diversity, and polymorphism information content were $0.11-0.91,0-0.90,0.17-0.94$, and 0.15-0.93, respectively. Interspecies transferability values were $93.5 \%, 91.6 \%$, and $91.1 \%$ for $G$. echinata, G. glabra, and G. uralensis, respectively. Phylogenetic analysis clustered cultivated (group 1) and wild (group 2) species into three and two subgroups, respectively. The reported markers represent a valuable resource for the genetic characteri $z$ ation of Glycyrrhiza spp. for theanalysis of its genetic variability, and as a tool for licorice transferability. This is the first intraspecific study in a collection of Glycyrrhiza spp. 


\section{Development of genomic simple sequence repeat}

2 markers for Glycyrrhiza lepidota and cross-

3 amplification of other Glycyrrhiza species

4

5

Jun Hyoung Bang ${ }^{1}$, Chi Eun Hong ${ }^{2}$, Sebastin Raveendar ${ }^{3}$, Kyong Hwan Bang ${ }^{2}$, Kyung $\mathrm{Ho} \mathrm{Ma}^{2}$, Soon Wook Kwon ${ }^{4}$, Hojin $\mathrm{Ryu}^{5}$, Ick Hyun Jo², Jong Wook Chung ${ }^{1}$

${ }^{1}$ Department of Industrial Plant Science and Technology, Chungbuk National University, Cheongju 28644, Republic of Korea

${ }^{2}$ Department of Herbal Crop Research, National Institute of Horticultural and Herbal Science, Rural Development Administration, Eumseong 27709, Republic of Korea

${ }^{3}$ National Agrobiodiversity Center, National Institute of Agricultural Science, RDA, Jeonju 54874, Republic of Korea

${ }^{4}$ Department of Plant Bioscience, Pusan National University, Miryang 50463, Republic of Korea

${ }^{5}$ Department of Biology, Chungbuk National University, Cheongju 28644, Republic of Korea

Corresponding Authors:

Ick Hyun $\mathrm{Jo}^{2}$, Jong Wook Chung ${ }^{1}$

292, Bisan-ro, Eumseong, Chungcheongbuk-do, 27709, Republic of Korea

${ }^{1} 1$, Chungdae-ro, Cheonju, Chungcheongbuk-do, 28644, Republic of Korea

Email address: intron@korea.kr (I. H. Jo); jwchung73@,chungbuk.ac.kr (J. W. Chung)

\section{Abstract}

Background. Licorice (Glycyrrhiza spp. L.) is used as a natural sweetener and medicinal herb in

European and Asian countries. Molecular studies have been conducted to find differences

between wild and cultivated species because most wild species are highly resistant to abiotic and biotic stresses compared with their cultivated species. However, few molecular markers have been developed for studying the genetic diversity and population structure of licorice species and to identify differences between cultivars. Thus, the present study aimed to develop a set of genomic simple sequence repeat (SSR) markers for molecular studies of these species.

Methods. In the present study, we developed polymorphic SSR markers based on whole-genome sequence data of Glycyrrhiza lepidota. Then, based on the sequence information, the 
33 polymorphic SSR markers were developed. The SSR markers were applied to 23 Glycyrrhiza

34 individual plants. We also evaluated the phylogenetic relationships and interspecies

35 transferability among samples.

37 Results. The genetic diversity analysis using these markers identified 2-23 alleles, and the major allele frequency, observed heterozygosity, genetic diversity, and polymorphism information content were $0.11-0.91,0-0.90,0.17-0.94$, and $0.15-0.93$, respectively. Interspecies

40

transferability values were $93.5 \%, 91.6 \%$, and 91.1\% for G. echinata, G. glabra, and G.

uralensis, respectively. Phylogenetic analysis clustered cultivated (group 1) and wild (group 2)

species into three and two subgroups, respectively. The reported markers represent a valuable resource for the genetic characterization of Glycyrrhiza spp. for the analysis of its genetic variability, and as a tool for licorice transferability. This is the first intraspecific study in a collection of Glycyrrhiza spp. germplasm using SSR markers.

Keywords : Cross-amplification, Genetic diversity, Licorice, Simple sequence repeat marker, Transferability

\section{Introduction}

The genus Glycyrrhiza L. (family Leguminosae) comprises approximately 20 recognized species distributed worldwide, most of which are perennial plants. The genus name Glycyrrhiza is derived from the ancient Greek word for 'sweet root' (Gr. glykos (sweet) + rhiza (root)), which was later Latinized to liquiritia and eventually to licorice (Isbrucker \& Burdock, 2006). Generally, licorice refers to the roots of Glycyrrhiza glabra, Glycyrrhiza uralensis, and Glycyrrhiza inflata (Jung et al., 2015). Licorice has been used as a medicinal herb to treat 
58 various diseases such as diabetes and depression. Glycyrrhizin, a triterpene found in the roots of 59 Glycyrrhiza spp., exhibits pharmacological activities, including anticancer, detoxification, and 60 anti-oxidant activities (Montoro et al., 2011). In addition, licorice root is a natural sweetener, and 61 it is used as a flavoring agent in food production worldwide (Snow, 1996; Gyawali et al., 2008; 62 Zhang and Ye, 2009). Licorice is sold in the form of slices or powder within Asian market, and 63 therefore, it is difficult to visually determine its variety and origin of production. Various studies 64 have been conducted to identify varieties to secure their sovereignty at the national level in 65 agreement with the Nagoya Protocol, an international treaty for sharing profits resulting from the 66 utilization of biological resources (Bang et al., 2011; Zhao et al., 2012).

67 Several types of molecular markers utilize variations in DNA sequences, including random 68 amplified polymorphic DNA, amplified fragment length polymorphism, single nucleotide 69 polymorphism, and simple sequence repeat (SSR) markers. The genomes of plants and 70 eukaryotic organisms contain a large number of SSRs (Hamada, Petrino \& Kakunaga, 1982; 71 Delseny, Laroche \& Penon,1983; Tautz \& Renz, 1984), which are widely distributed in the 72 coding and non-coding regions of nuclear and organellar DNA (Vieira et al., 2016). The SSR 73 markers are co-dominant and have higher polymorphism and reproducibility than other DNA 74 markers. Therefore, they are broadly used for identifying species and cultivars, as well as for 75 analyzing genetic diversity and population structure (Kalia et al., 2011; Ya et al., 2017). Because 76 SSR markers can be transferred between species (Lichtenzveig et al., 2005), they are suitable not 77 only for determining genetic diversity but also for cross-species amplification, which reduces the 78 cost and time associated with such analyses.

79 Wild species usually present higher resistance to abiotic and biotic stresses compared to 80 cultivated plant species, and some lineages have desirable genetic traits. Genetic studies have 
81 been conducted on wild and cultivated species of crops such as potato and tomato (Rick \&

82 Chetelat, 1995; Singh, Ocampo \& Robertson, 1998). Similarly, Ashurmetov (1996) examined the

83 genetic relationships between wild and cultivated species of licorice. Recently, SSR markers

84 were developed for licorice species based on the chloroplast genomes of G. uralensis, which is a

85 cultivated species (Liu et al., 2015), and G. lepidota, which is a wild species (Raveendar et al.,

86 2017; Jo et al., 2018); on the transcriptome of G. uralensis and G. glabra, also a cultivated

87 species (Um et al., 2016); and on the nuclear genome of G. lepidota (Lee et al., 2019). However,

88 to the best of our knowledge, molecular studies on genomic SSR markers of licorice have not

89 been conducted. Thus, the present study aimed to develop genomic SSR markers for licorice

90 molecular genetic studies, including the differentiation between wild and cultivated species, their

91 genetic diversity, and population structure.

92

93

Materials \& Methods

94

95

Plant material and DNA isolation

96 The materials used in the present study comprised 11 accessions of G. uralensis and G. glabra

97 obtained from the Ginseng Research Division at the National Institute of Horticultural and

98 Herbal Science (NIHHS), South Korea, and 12 accessions obtained from the United States

99 Department of Agriculture (USDA), including G. uralensis, G. glabra, G. lepidota, G. echinata,

100 and Glycyrrhiza spp. (Table 1). Seedlings of each licorice accession were grown in pots of sterile

101 soil in a greenhouse with three plants per accession. Leaf samples were purposively sampled

102 from three plants per accession from 4-week-old seedlings and ground to powder using liquid

103 nitrogen in a pestle-mortar; their genomic DNA was extracted using the Plant gDNA Extraction

104 Kit (GeneAll, Seoul, Korea) following the manufacturer's protocol. 
106 Primer design and polymerase chain reaction

107 In our previous study, the chloroplast genome of G. lepidota (NCBI accession no. KY038482)

108 was obtained by the de novo assembly of the low-coverage whole-genome sequence via a

109 bioinformatics pipeline (http://phyzen.com) (Raveendar et al., 2018). We mined SSRs based on a

110 whole-genome sequence that was not used in the chloroplast genome analysis of G. lepidota. The

111 whole-genome sequence of G. lepidota were searched for SSRs using the MIcroSAtellite (MISA,

112 http://pgrc.ipk-gatersleben.de/misa/) with the search criteria for minimum number of repeats set

113 at six for mono-nucleotide repeats, five for di-nucleotide repeats, four for tri-nucleotide repeats,

114 and three each for tetra-, penta-, hexa-, hepta-, octa-, nona- and deca-nucleotide motifs. Next,

115100 SSR primer pairs with di- and tri-nucleotide repeat motifs were randomly designed using

116 Primer 3.0 (http://primer3.sourceforge.net/) with the following criteria: primer length (18 - $23 \mathrm{bp}$,

117 with optimum value $20 \mathrm{bp})$; $\operatorname{Tm}\left(54^{\circ} \mathrm{C}-56^{\circ} \mathrm{C}\right.$, with optimum value $\left.55^{\circ} \mathrm{C}\right)$; $\mathrm{GC}$ content $(40 \%$ -

$11860 \%$, with the optimum value 50\%); maximum Tm difference between forward and reverse

119 primer $1.5^{\circ} \mathrm{C}$ and product size range (100 - $350 \mathrm{bp}$ with optimum value $\left.250 \mathrm{bp}\right)$. The first

120 polymerase chain reaction (PCR) using all the primer sets was performed for four accessions of

121 G. lepidota (CBG20-23) to establish the PCR conditions. The primers resulting in successful

122 amplifications were then applied to assess the genetic diversity of the 23 Glycyrrhiza spp.

123 accessions. The PCR mixture (total volume, $40 \mu \mathrm{L}$ ) contained 20 ng genomic DNA, 10 pmol

124 each primer, $2.5 \mathrm{mM} \mathrm{MgCl} 2,0.25 \mathrm{mM}$ dNTPs, and 0.5 U Taq polymerase (Inclone, Deajeon,

125 Korea). The amplification was performed on a CFX96 PCR detection system (Bio-Rad

126 Laboratories, Hercules, CA, USA) and included 30 cycles of pre-denaturation at $94{ }^{\circ} \mathrm{C}$ for 5 min,

127 denaturation at $94{ }^{\circ} \mathrm{C}$ for $30 \mathrm{~s}$, annealing at $55-60{ }^{\circ} \mathrm{C}$ for $45 \mathrm{~s}$, and extension at $72{ }^{\circ} \mathrm{C}$ for $1 \mathrm{~min}$. 
128 The PCR products were separated and visualized using a Fragment Analyzer (Agilent

129 Technologies, Santa Clara, CA, USA).

130

131 Data analyses

132 The number of alleles, major allele frequency, genetic diversity, observed heterozygosity, and 133 polymorphism information content (PIC) were analyzed using PowerMarker 3.25

134 (https://brcwebportal.cos.ncsu.edu/powermarker/). The rate of cross-amplification

135 (transferability) among the 23 licorice accessions was measured using the following equation:

136 Transferability $(\%)=$ amplicons (bands amplified by PCR) $\times 100 /$ theoretical amplicons (primer

137 number $\times$ sample size) (Lee et al., 2015; Raveendar et al., 2015). Phylogenetic analysis was

138 performed using the Cavalli-Sforza chord distance (Cavalli-Sforza \& Edwards, 1967) included in

139 PowerMarker; the phylogenetic tree was constructed in MEGA4 (Tamura et al., 2007) using the

140 unweighted pair group method with arithmetic mean.

141

142 Results

143

144 Diversity of SSR markers and interspecies cross-amplification

145 From the whole-genome sequence of G. lepidota, 249,492 SSRs were mined. The frequency

146 distribution of mined SSR repeat types is presented in Table S1. It was observed that

147 mononucleotide repeats $(218,320 ; 87.5 \%)$ were the most abundant repeat type, followed by

148 dinucleotide repeats $(11,056 ; 4.4 \%)$, tetra-nucleotide repeats $(9365 ; 3.8 \%)$, tri-nucleotide repeats

$149(7580 ; 3 \%)$, penta-nucleotide repeats $(3170 ; 1.3 \%)$, and hexa-nucleotide repeats $(1387 ; 0.6 \%)$ in

150 G. lepidota whole-genome, respectively (Table S1). 
151 Sixty-two of the 100 selected primers amplified all the four accessions of G. lepidota (CBG20-

152 23). Thus, these 62 primers were used to analyze the diversity of the 23 licorice accessions,

153 which resulted in the identification of 549 alleles; the number of alleles per marker ranged from

1542 (GL-gSSR-019) to 23 (GL-gSSR-028), with a mean of 9.6 alleles. The major allele frequency

155 ranged from 0.11 (GL-gSSR-088) to 0.91 (GL-gSSR-019), with a mean of 0.349. The observed

156 heterozygosity ranged from 0 (GL-gSSR-006, -019, -023, -068, -090, -097, and -100) to 0.70

157 (GL-gSSR-095), with a mean of 0.264. The maximum values of genetic diversity and PIC,

158 indicating the genetic diversity of the markers, were 0.94 and 0.93 , respectively, in GL-gSSR-

159 028, whereas the minimum values were 0.17 and 0.15 , respectively, in GL-gSSR-019. The mean

160 values of genetic diversity and PIC were 0.760 and 0.730 , respectively (Table S2). Cross-

161 amplification analysis among licorice species revealed that $G$. echinata had the highest

162 transferability (93.5\%), followed by G. glabra (91.6\%) and G. uralensis $(91.1 \%)$; the mean value 163 was $92.07 \%$ (Table 2).

164

165 Phylogenetic analysis

166 The 23 accessions were classified into two clusters (Fig. 1), one corresponding to the cultivated 167 plant species (group 1) and the other to the wild species (group 2). The 15 accessions within 168 group 1 were arranged into three subgroups: group 1-1 contained two G. glabra accessions; 169 group 1-2 contained six G. uralensis accessions, one G. glabra accession, and one Glycyrrhiza 170 sp. accession; and group 1-3 contained two G. glabra accessions and three Glycyrrhiza sp.

171 accessions. The seven accessions within group 2 were arranged into two subgroups, groups 2-1

172 and 2-2, which contained six G. lepidota and two G. echinata accessions, respectively. 


\section{Discussion}

175

176 Diversity of the SSR markers

177 The mean PIC value of the SSR markers developed in the present study using wild (G. lepidota

178 and G. echinata) and cultivated (G. uralensis and G. glabra) licorice accessions was 0.730,

179 which is higher than that reported for other medicinal plants: 0.314 for Zingiber officinale

180 (Pandotra et al., 2013), 0.57 for Codonopsis lanceolata (Kim et al., 2016), and 0.272 for Festuca

181 arundinacea (Tehrani et al., 2009). These results showed that the SSR markers developed in the

182 present study have a higher diversity than those developed for other species. Moreover, Um et al.

183 (2016) reported the PIC value of 0.56 for eight SSR markers based on G. uralensis genome using

18422 accessions of cultivated licorice species ( $G$. uralensis and G. glabra). To compare the

185 markers developed in the present study with those previously reported, the PIC values of the 11

186 accessions of cultivated licorice species (G. uralensis and G. glabra) were measured, and a mean

187 value of 0.624 was obtained. Thus, the SSR markers developed in the present study had a higher

188 mean PIC value than those previously developed for G. uralensis, despite the lower number of

189 accessions and higher number of primers used here. This might be attributed to the fact that the

190 primers with PIC value higher than 0.63 accounted for $66.13 \%$ of the total primers. These results

191 suggested that the markers developed in the present study should be more efficient for molecular

192 genetic studies than those reported in previous studies due to their higher diversity.

193 In the diversity analysis based on repeat motifs, the mean PIC values of dinucleotide repeat

194 motifs (32 sets of primers) and trinucleotide repeat motifs (30 sets of primers) were 0.796 and

195 0.688, respectively, showing a higher value for the dinucleotide repeat motifs. The higher mean

196 PIC value for dinucleotide repeats might be related to the fact that dinucleotide repeats are 
197 distributed throughout the genome, whereas trinucleotide repeats are mostly present in coding

198 regions. Furthermore, trinucleotide repeats are under a relatively weaker selection pressure

199 against mutations that alter the reading frame compared with the dinucleotide repeats, leading to

200 a lower genetic diversity in the trinucleotide repeats (Kalia et al., 2011; Vieira et al., 2016).

201 Considering that dinucleotide repeats had higher PIC values than trinucleotide repeats, despite

202 the similar number of primers, the primers based on dinucleotide repeats are expected to be more 203 useful for studies regarding diversity analysis.

204

205 Interspecies cross-amplification

206 The mean interspecies transferability of licorice was $92.5 \%$. The mean interspecies

207 transferabilities of Rubus coreanus and Allium sativum were 73.52\% (Lee et al., 2015) and

$20858.85 \%$ (Lee et al., 2011), respectively, and that of Triticum aestivum and Secale cereale were

$20948.4 \%$ and 35.3\%, respectively (Kuleung, Baenziger \& Dweikat, 2004). According to the report

210 of Erayman et al. (2014), the transferabilities of SSR markers developed for Medicago

211 truncatula, Phaseolus vulgaris, and Cicer arietinum to G. glabra, G. echinata, and G. flavescens

212 were $33 \%$ for M. truncatula, 11\% for P. vulgaris, and 6\% for C. arietinum. These results

213 indicated that the markers developed for Glycyrrhiza accessions in the present study had higher

214 transferability than those developed for other species. Interspecies crossing rates between $G$.

215 lepidota and other licorice species were 51-75\% (Ashurmetov, 1996), suggesting that crossing

216 may occur under natural conditions, and genes of G. lepidota could affect other licorice species.

217 We postulated that these are the likely reasons why interspecies transferability has become

218 relatively high. In particular, G. lepidota had a high transferability with G. echinata, another wild

219 species, possibly because these are more closely related to each other than to cultivated species. 
220 Such results showed that the SSR markers developed for the wild species G. lepidota can be

221 efficiently used for other licorice species. However, as the number of resources used in the

222 present study was smaller than that in other studies, future studies should include various species.

223

224 Phylogenetic analysis of licorice accessions

225 In the present study, although CBG4 and CBG6 (G. glabra accessions) clustered with CBG16 226 and CBG17 (also G. glabra accessions), they were separated into groups 1-1 and 1-3,

227 respectively. Such clustering could be due to the random selection of markers. In addition, the 228 separation of G. glabra into two subgroups might be due to their genetic differences arising from 229 their production origins. The four Glycyrrhiza spp. accessions CGB08, CGB09, CGB10, and 230 CGB11 were separated into two groups, suggesting that CBG09 is genetically closer to $G$. 231 uralensis, whereas CBG08, CGB10, and CGB11 are genetically closer to G. glabra. Accession

232 CBG3 (G. glabra) clustered with G. uralensis, which might be due to either incomplete 233 identification of resources during collection or insufficient numbers of licorice accessions (Jo et 234 al., 2018; Lee et al., 2019). However, Jo et al. (2018) estimated that the wild species G. echinata 235 was genetically close to the cultivated species G. uralensis.

236

\section{Conclusion}

238 The clustering pattern obtained in the present study was congruent with the results of Lee et al. 239 (2019), indicating that the SSR markers that allow the analysis of intra-species diversity might 240 also be efficient for interspecies differentiation. Moreover, the SSR markers developed in the 241 present study might be successfully applied in molecular genetic studies aiming to differentiate 242 wild and cultivated licorice and to determine their diversity. 


\section{Acknowledgements}

245 The authors thank Dr. Lee (Department of Herbal Crop Research, National Institute of

246 Horticultural and Herbal Science, Rural Development Administration, Eumseong, Republic of

247 Korea) for his assistance in collecting the licorice sample.

248

249 Competing Interests

250 The authors declare that they have no competing interests.

251

\section{Author Contributions}

253 Jun Hyoung Bang conceived and designed the experiments, performed the experiments, analyzed 254 the data, prepared figures and/or tables, authored or reviewed drafts of the paper, approved the 255 final draft.

256 Chi Eun Hong and Sebastin Raveendar conceived and designed the experiments, contributed 257 reagents/materials/analysis tools, approved the final draft.

258 Kyong Hwan Bang, Kyung Ho Ma, Soon Wook Kwon and Hojin Ryu contributed 259 reagents/materials/analysis tools, approved the final draft.

260 Ick Hyun Jo and Jong Wook Chung analyzed the data, contributed reagents/materials/analysis 261 tools, prepared figures and/or tables, authored or reviewed drafts of the paper, approved the final 262 draft.

263

264 References 
266 Ashurmetov O. 1996. Selection of parental pairs for obtaining hybrids in the genera Glycyrrhiza 267 L. and Meristotropis Fisch. et Mey. Genet Resour Crop Evol 43:167-171 DOI:

268

269

270

271

272

273

274

275

276

277

278

279

280

281

282

283

284

285

286

287 https://doi.org/10.1007/BF00126760.

Bang KH, Chung JW, Kim YC, Lee JW, Jo IH, Seo AY, Kim OK, Hyun DY, Kim DH, Cha SW. 2011. Development of SSR markers for identification of Korean ginseng (Panax ginseng CA Meyer) cultivars. Korean J Med Crop Sci 19:185-190 DOI: 10.5142/jgr.2011.35.4.504.

Cavalli-Sforza LL, Edwards AW. 1967. Phylogenetic analysis: models and estimation procedures. Evolution 21:550-570.

Delseny M, Laroche M, Penon P. 1983. Detection of sequences with Z-DNA forming potential in higher plants. Biochem Biophy Res Commun 116:113-120.

Erayman M, Ilhan E, Guzel Y, Eren AH. 2014. Transferability of SSR markers from distantly related legumes to Glycyrrhiza species. Turk J Agric For 38:32-38 DOI: 10.3906/tar-130347.

Gyawali R, Seo HY, Shim SL, Ryu KY, Kim W, You SG, Kim KS. 2008. Effect of $\gamma$-irradiation on the volatile compounds of licorice (Glycyrrhiza uralensis Fischer). Eur Food Res Technol 226:577-582 DOI: 10.1007/s00217-007-0591-2.

Hamada H, Petrino MG, Kakunaga T. 1982. A novel repeated element with Z-DNA-forming potential is widely found in evolutionarily diverse eukaryotic genomes. Proc. Natl. Acad. Sci. USA 79:6465-6469.

Isbrucker RA, Burdock GA. 2006. Risk and safety assessment on the consumption of Licorice root (Glycyrrhiza sp.), its extract and powder as a food ingredient, with emphasis on the 
288

289

290

291

292

293

294

295

296

297

298

299

300

301

302

303

304

305

306

307

308

pharmacology and toxicology of glycyrrhizin. Regul. Toxicol. Pharm. 46:167-192 DOI: https://doi.org/10.1016/j.yrtph.2006.06.002.

Jo IH, Sung J, Hong CE, Raveendar S, Bang KH, Chung JW. 2018. Development of cleaved amplified polymorphic sequence (CAPS) and high-resolution melting (HRM) markers from the chloroplast genome of Glycyrrhiza species. 3 Biotech 8:220. DOI: $10.1007 / \mathrm{s} 13205-018-1245-8$.

Jung JC, Lee YH, Kim SH, Kim KJ, Kim KM, Oh S, Jung YS. 2015. Hepatoprotective effect of licorice, the root of Glycyrrhiza uralensis Fischer, in alcohol-induced fatty liver disease. BMC Complement Altern Med 16:19 DOI: 10.1186/s12906-016-0997-0.

Kalia RK, Rai MK, Kalia S, Singh R, Dhawan AK. 2011. Microsatellite markers: an overview of the recent progress in plants. Euphytica 177:309-334 DOI: https://doi.org/10.1007/s10681010-0286-9.

Kim S, Jung JH, Chung H, Kim JH, Gil J, Yoo J, Um Y, Kim OT, Kim TD, Kim YY, Lee DH, Kim HB, Lee Y. 2016. Simple sequence repeat marker development from Codonopsis lanceolata and genetic relation analysis. J Plant Biotechnol 43:181-188 DOI: 10.5010/JPB.2016.43.2.181.

Kuleung C, Baenziger PS, Dweikat I. 2004. Transferability of SSR markers among wheat, rye, and triticale. Theor Appl Genet 108:1147-1150 DOI: 10.1007/s00122-003-1532-5.

Lee GA, Kwon SJ, Park YJ, Lee MC, Kim HH, Lee JS, Lee SY, Gwag JG, Kim CK, Ma KH. 2011. Cross-amplification of SSR markers developed from Allium sativum to other Allium species. Sci Hort 128:401-407. 
309 Lee GA, Song J, Choi HR, Chung JW, Jeon YA, Lee JR, Ma KH, Lee MC. 2015. Novel

310 microsatellite markers acquired from Rubus coreanus Miq. and cross-amplification in other 311 Rubus Species. Molecules 20:6432-6442.

312 Lee KJ, Raveendar S, Choi JS, Gil J, Lee JH, So YS, Chung JW. 2019. Development of 313 chloroplast microsatellite markers for identification of Glycyrrhiza species. Plant Genet $314 \quad$ Resour 17:95-98 DOI: 10.1017/S1479262118000308.

315 Lichtenzveig J, Scheuring C, Dodge J, Abbo S, Zhang HB. 2005. Construction of BAC and 316 BIBAC libraries and their applications for generation of SSR markers for genome analysis 317 of chickpea, Cicer arietinum L. Theor Appl Genet 110:492-510 DOI: 10.1007/s00122$318 \quad 004-1857-8$.

319 Liu Y, Zhang P, Song M, Hou J, Qing M, Wang W, Liu C. 2015. Transcriptome analysis and 320 development of SSR molecular markers in Glycyrrhiza uralensis Fisch. PLOS ONE 321 10:e0143017 DOI: 10.1371/journal.pone.0143017.

322

323

324

325

326

327

328

329

330

Montoro P, Maldini M, Russo M, Postorino S, Piacente S, Pizza C. 2011. Metabolic profiling of roots of liquorice (Glycyrrhiza glabra) from different geographical areas by ESI/MS/MS and determination of major metabolites by LC-ESI/MS and LC-ESI/MS/MS. J Pharm Biomed Anal 54:535-544 DOI: 10.1016/j.jpba.2010.10.004.

Pandotra P, Gupta AP, Husain MK, Gupta S. 2013. Evaluation of genetic diversity and chemical profile of ginger cultivars in north-western Himalayas. Biochem Syst Ecol 48:281-287.

Raveendar S, Lee GA, Jeon YA, Lee Y, Lee JR, Cho GT, Cho JH, Park JH, Ma KH, Chung JW. 2015. Cross-amplification of Vicia sativa subsp. sativa microsatellites across 22 other Vicia species. Molecules 20:1543-1550 DOI: 10.3390/molecules20011543. 
331 Raveendar S, So YS, Lee KJ, Lee DJ, Sung J, Chung JW. 2017. The complete chloroplast 332 genome sequence of Glycyrrhiza lepidota (Nutt.) Pursh - An American wild licorice. J 333 Crop Sci Biotechnol 20:295-303.

334 Rick CM, Chetelat RT. 1995. Utilization of related wild species for tomato improvement. In:

335 Fernández-Muñoz R, Cuartero J, Gómez-Guillamón ML (eds) International Symposium on $336 \quad$ Solanaceae for Fresh Market. Acta Hortic 412:21-38.

337 Singh KB, Ocampo B, Robertson LD. 1998. Diversity for abiotic and biotic stress resistance in 338 the wild annual Cicer species. Genet Resour Crop Evol 45:9-17 DOI:

339 https://doi.org/10.1023/A:1008620002136.

340 Snow J. 1996. Glycyrrhiza glabra monograph. J Bot Med 1:9-14.

341 Tamura K, Dudley J, Nei M, Kumar S. 2007. MEGA4: molecular evolutionary genetics analysis 342 (MEGA) software version 4.0. Mol Biol Evol 24:1596-1599 DOI: 10.1093/molbev/msm092.

Tautz D, Renz M. 1984. Simple sequences are ubiquitous repetitive components of eukaryotic genomes. Nucleic Acids Res 12:4127-4138.

346 Tehrani MS, Mardi M, Sahebi J, Catalan P, Diaz-Perez A. 2009. Genetic diversity and structure among Iranian tall fescue populations based on genomic-SSR and EST-SSR marker analysis. Plant Syst Evol 282:57-70 DOI: https://doi.org/10.1007/s00606-009-0207-3.

Um Y, Jin ML, Lee Y, Hur M, Cha SW, Jung CS, Kim SM, Lee JH. 2016. Genetic diversity 350 analysis of Glycyrrhiza uralensis using 8 novel polymorphic microsatellite markers. $J$ Plant Biotechnol 43:174-180 DOI: http://dx.doi.org/10.5010/JPB.2016.43.2.174. 
352 Vieira MLC, Santini L, Diniz AL, Munhoz CDF. 2016. Microsatellite markers: what they mean 353 and why they are so useful. Genet Mol Biol 39:312-328 DOI: 10.1590/1678-4685-GMB$354 \quad 2016-0027$.

355 Ya N, Raveendar S, Bayarsukh N, Ya M, Lee JR, Lee KJ, Shin MJ, Cho GT, Ma KH, Lee GA. 356 2017. Genetic diversity and population structure of Mongolian wheat based on SSR 357 markers: Implications for conservation and management. Plant Breed Biotech 5:213-220 DOI: https://doi.org/10.9787/PBB.2017.5.3.213

Zhang Q, Ye M. 2009. Chemical analysis of the Chinese herbal medicine Gan-Cao (licorice). $J$ 360 Chromatogr A 1216:1954-1969 DOI: 10.1016/j.chroma.2008.07.072.

361 Zhao Y, Williams R, Prakash CS, He G. 2012. Identification and characterization of gene-based SSR markers in date palm (Phoenix dactylifera L.). BMC Plant Biol 12:237 DOI: $10.1186 / 1471-2229-12-237$. 
Figure 1

Phylogenetic tree of the 23 Glycyrrhiza sp. accessions based on 62 simple sequence repeat markers developedhere, as well as on the Cavalli-Sforza chord genetic distance.

G. uralensis

G. glabra

G. sp.

G. lepidota

G. echinata

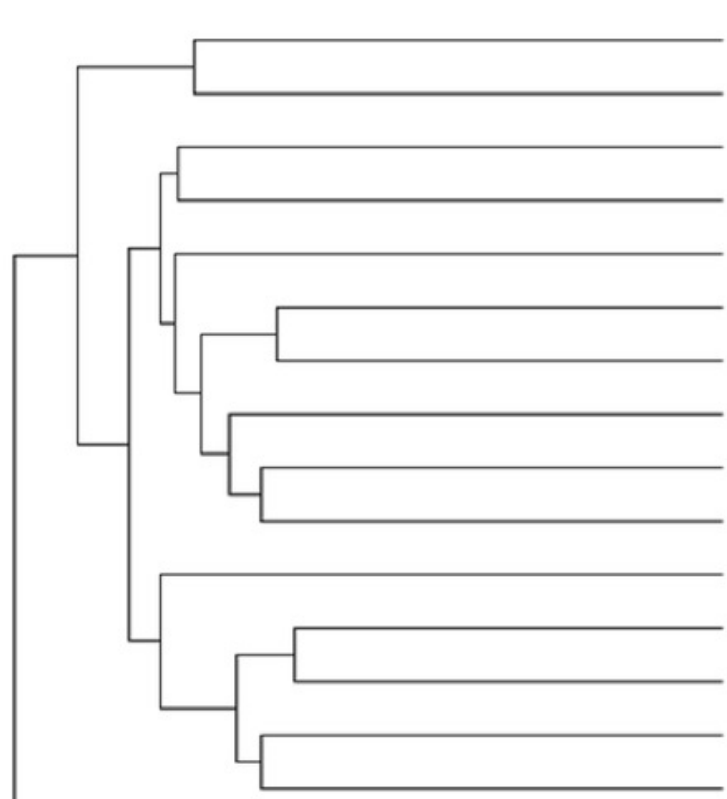

CBG16-KGZ CBG17-KGZ CBG01-CAN CBG09-KOR CBG05-MNG CBG14-KAZ CBG15-KGZ CBG07-CHN CBG02-RUS CBG03-CHN CBG04-CAN CBG08-KOR CBG11-KOR CBG06-UZB CBG10-KOR

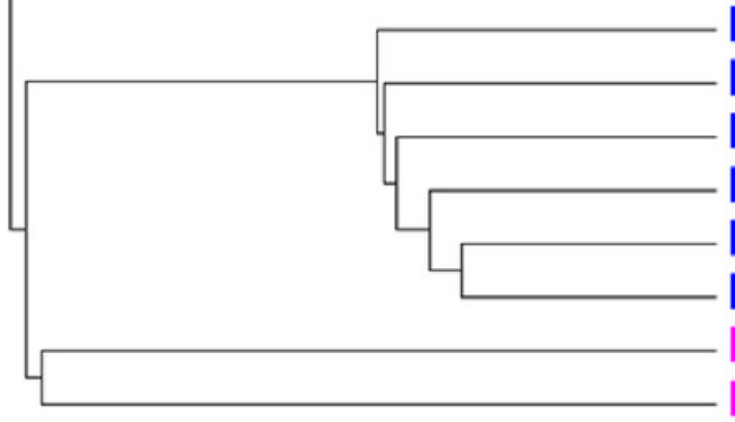
CBG18-USA CBG19-USA - CBG23-USA CBG13-DEU
Group1-1

Group 1-2

G

R

$\mathrm{O}$

U

$\mathrm{P}$

1

Groupl-3

Group2-2

Group2-1
G

$\mathrm{R}$

$\mathrm{O}$

2 CBG20-USA CBG21-USA CBG22-USA CBG12-YUG

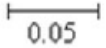




\section{Table 1 (on next page)}

Glycyrrhiza sp. accessions used in the present study. 
1 Table 1 Glycyrrhiza sp. accessions used in the present study

\begin{tabular}{llll}
\hline Accession code & Species & Origin $^{\mathbf{a}}$ & Institution $^{\mathbf{b}}$ \\
\hline CBG1 & Glycyrrhiza uralensis & CAN & NIHHS \\
CBG2 & G. uralensis & RUS & NIHHS \\
CBG3 & Glycyrrhiza glabra & CHN & NIHHS \\
CBG4 & G. glabra & CAN & NIHHS \\
CBG5 & G. uralensis & MNG & NIHHS \\
CBG6 & G. glabra & UZB & NIHHS \\
CBG7 & G. uralensis & CHN & NIHHS \\
CBG8 & Glycyrrhiza spp. & KOR & NIHHS \\
CBG9 & Glycyrrhiza spp. & KOR & NIHHS \\
CBG10 & Glycyrrhiza spp. & KOR & NIHHS \\
CBG11 & Glycyrrhiza spp. & KOR & NIHHS \\
CBG12 & Glycyrrhiza echinata & YUG & USDA ARS \\
CBG13 & G. echinata & DEU & USDA ARS \\
CBG14 & G. uralensis & KAZ & USDA ARS \\
CBG15 & G. uralensis & KGZ & USDA ARS \\
CBG16 & G. glabra & KGZ & USDA ARS \\
CBG17 & G. glabra & KGZ & USDA ARS \\
CBG18 & Glycyrrhiza lepidota & USA & USDA ARS \\
CBG19 & G. lepidota & USA & USDA ARS \\
CBG20 & G. lepidota & USA & USDA ARS \\
CBG21 & G. lepidota & USA & USDA ARS \\
CBG22 & G. lepidota & USA & USDA ARS \\
CBG23 & G. lepidota & USA & USDA ARS \\
\hline
\end{tabular}

2 a Refers to the region where the specimen was collected: CAN, Canada; RUS, Russia; CHN, China; MNG, Mongolia; UZB,

3 Uzbekistan; KOR, Korea; YUG, Yugoslavia; DEU, Germany; KAZ, Kazakhstan; KGZ, Kyrgyzstan; USA, United States of

4 America

$5{ }^{\mathrm{b}}$ Refers to the institution where the accessions are deposited: NIHHS, National Institute of Horticultural and Herbal

6 Science; USDA ASR: United States Department of Agriculture Agricultural Research Service 


\section{Table 2 (on next page)}

Transferability of the 62 Glycyrrhiza lepidota simple sequence repeat (SSR) markers to other Glycyrrhiza species. 
1 Table 2 Transferability of the 62 Glycyrrhiza lepidota simple sequence repeat (SSR) markers to

2 other Glycyrrhiza species

\begin{tabular}{|c|c|c|c|}
\hline $\begin{array}{l}\text { Species } \\
\text { (Sample size) }\end{array}$ & $\begin{array}{c}\text { Theoretical amplicons } \\
\text { (number) }\end{array}$ & $\begin{array}{c}\text { Amplicons } \\
\text { (number) }\end{array}$ & $\begin{array}{c}\text { Transferability } \\
(\%)\end{array}$ \\
\hline $\begin{array}{l}\text { Glycyrrhiza uralensis } \\
\text { (6) }\end{array}$ & 372 & 339 & 91.1 \\
\hline $\begin{array}{l}\text { Glycyrrhiza glabra } \\
\text { (5) }\end{array}$ & 310 & 284 & 91.6 \\
\hline $\begin{array}{l}\text { Glycyrrhiza echinata } \\
\text { (2) }\end{array}$ & 124 & 116 & 93.5 \\
\hline Mean & & 268.6 & 92.07 \\
\hline
\end{tabular}

3 